\title{
Calculation of Muscle and Joint Forces in the Masticatory System
}

\author{
S. Rues, H.J. Schindler, J. Lenz, K. Schweizerhof \\ Universität Karlsruhe, Forschungsgruppe Biomechanik
}

2006

Institut für Mechanik

Kaiserstr. 12, Geb. 20.30

76128 Karlsruhe

Tel.: +49 (0) 721/ 608-2071

Fax: +49 (0) 721/ 608-7990

E-Mail: ifm@uni-karlsruhe.de

www.ifm.uni-karlsruhe.de 


\title{
Calculation of Muscle and Joint Forces in the Masticatory System
}

\author{
S. Rues, H. J. Schindler, J. Lenz \& K. Schweizerhof \\ Research Group Biomechanics, University of Karlsruhe \\ 76128 Karlsruhe, Germany
}

\begin{abstract}
In ten healthy test persons, electromyographic (EMG) activities of the essential masticatory muscles and the intraorally transferred, feedback-controlled resultant bite force have been measured simultaneously for 19 motor tasks at different magnitudes simulating clenching. Additionally, for all test persons 3D-models of the musculature were reconstructed from magnetic resonance tomograms. The aim of the study was to identify the associated activation patterns, the intrinsic muscle strength and the muscle forces using a non-linear force law, and to calculate the joint reaction forces. On the basis of this information, motor tasks leading to high joint forces may possibly be identified. However, for the calculation of joint forces the lines of action of the masticatory muscles and the magnitudes of the muscle forces are needed. In this contribution the lines of action are determined by two different schemes: In the first approach the lines of action are defined by the centroids of the muscles' origin and insertion areas, and the muscle force magnitudes are computed based on the physiological cross-sectional areas. In a second approach all quantities shall be computed with the help of specific finite elements which are presently under development.
\end{abstract}

\section{Introduction}

The human masticatory system consists of twelve essential muscles connecting the mandible with the maxilla. Each muscle can generate a force vector with an a priori unknown magnitude, but along a line of action which can approximately be constructed either from its geometry or with the help of a finite element (FE) analysis. For the joint forces, however, the magnitudes as well as the lines of action are unknown. If we assume the mandible to be a rigid body there are 12 muscle forces +6 joint force components in comparison to 6 equilibrium equations. Therefore, the system is highly redundant, i.e., without further information a specific resultant force can be generated by an infinite variety of activation patterns. Thus, aside of certain optimization methods using arbitrarily chosen target functions, only a simultaneous measurement of all muscular EMG activities and the resultant bite force between the lower and upper jaw can reveal the actual situation.

It is obvious that the quality of the results depends essentially on the quality of the estimation of the lines of action. Here, a FE analysis will certainly deliver better results than the geometric approach because the structure of each muscle and a possible inhomogeneous activation can be taken into account. 


\section{Materials and Methods}

\subsection{EMG- and Bite Force Measurement}

To date, no measured data are available from experiments in which the activities of all masticatory muscles and the resultant bite force have been recorded simultaneously. This complete knowledge is, however, indispensable to determine the direction and magnitude of the reaction forces transferred to the condyles.

For that purpose, in ten healthy male subjects (average age: $29 \pm 2.6$ years) the intraoral force transfer and the electromyographic activities of the masseter, anterior and posterior temporal, medial and lateral pterygoid, and anterior digastric were simultaneously recorded in simulated clenching tasks during the generation of various resultant bite force vectors $F_{\text {res. }}$ A feedback system enabled the test persons to perform 19 specific clenching tasks (circumferential angle $\varphi=0^{\circ}, 60^{\circ}, 90^{\circ}, 180^{\circ}, 270^{\circ}, 300^{\circ}$; cranial angle $\theta=0^{\circ}$, $20^{\circ}, 40^{\circ}, 60^{\circ}$ with respect to the normal z' on the occlusal plane; cf. Fig. 1 , left) at different magnitudes of the resultant force. The centrally transmitted resultant force was determined with an intraoral measuring appliance, consisting of a bearing pin device equipped with strain gauges and fixed on custom-made metal splints (Fig. 1, middle).
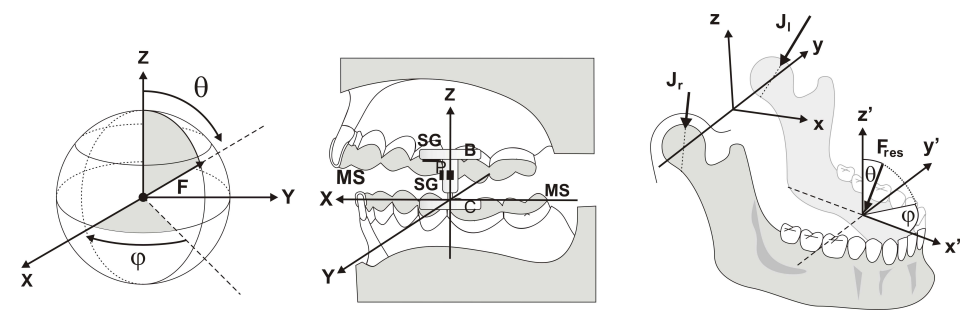

Fig. 1. Left: Coordinate system used for the force measurement device (x',y'-plane corresponds to the occlusal plane) with angles $\varphi$ and $\theta$. Middle: Intraoral measuring device: SG: strain gauge, P: pin, B: base plate, C: contact plate, MS: metal splint. Right: Mandible with bite force and joint forces (muscle forces are not displayed).

Bipolar surface electrodes were employed to measure bilaterally the electric activities $\mathrm{U}_{\mathrm{i}}$ of the masseter, anterior temporal, posterior temporal, and anterior digastric, whereas bilateral bipolar wire electrodes, inserted by a needle, recorded the electric muscle activity of the medial and lateral pterygoid muscles. For special motor tasks also the maximum electric activities $U_{\max , i}$ of all muscles were determined. The experimental details are described in [1] . The study was approved by the Ethics Committee of the University of Freiburg, Germany (No. 25/02). All participating subjects gave their written consent to the experiments which were conducted in accordance with the Declaration of Helsinki. 


\subsection{Force Law}

In addition, for each test person a 3D-model of the musculature was constructed using horizontal and frontal magnetic resonance tomograms (MRT) which also served to identify the so-called Frankfurt horizontal plane (passing through the lowest point in the margin of the orbit and the highest point in the margin of the auditory meatus), the occlusal plane, and the position of the bearing pin. From these models the so-called physiological cross-sectional areas $\mathrm{A}_{\mathrm{i}}=\left(1-\mathrm{p}_{\mathrm{i}}\right) \mathrm{V}_{\mathrm{i}} / \mathrm{l}_{\mathrm{f}, \mathrm{i}}\left(\mathrm{V}_{\mathrm{i}}\right.$ : total muscle volume, $\mathrm{p}_{\mathrm{i}}$ : portion of tendinous tissue, $l_{f, i}$ : muscle fiber length) were calculated (cf. Fig. 2, left). The values for $\mathrm{V}_{\mathrm{i}}$ and $\mathrm{l}_{\mathrm{f}, \mathrm{i}}$ were taken from [2]. All described motor tasks were performed with magnitudes $\mathrm{F}_{\text {res }}=50 \mathrm{~N}$ and $\mathrm{F}_{\text {res }}=150 \mathrm{~N}$. The task with vertical resultant force was additionally performed with $\mathrm{F}_{\text {res }}=250 \mathrm{~N}$ and under maximum voluntary bite force of each test person. The results of this experimental study were presented in detail in [1]. The correlation between the actual muscle force and the actual electric activation is given by the force law:

$$
\frac{\mathrm{F}_{\mathrm{i}}}{\mathrm{F}_{\max , \mathrm{i}}}=f\left(\frac{\mathrm{U}_{\mathrm{i}}}{\mathrm{U}_{\max , \mathrm{i}}}\right)=\mathrm{c}_{1} \frac{\mathrm{U}_{\mathrm{i}}}{\mathrm{U}_{\max , \mathrm{i}}}+\mathrm{c}_{2}\left(\frac{\mathrm{U}_{\mathrm{i}}}{\mathrm{U}_{\max , \mathrm{i}}}\right)^{2}
$$

Using the measured data, the constants $c_{1}$ and $c_{2}$ were determined for each test person separately via a least squares fit. A typical result for one test person can be seen in Fig. 2, right. The muscle force is proportional to its physiological cross-section (= sum of all muscle fiber cross-sections) and the stress generated by the muscle fibers [3]. The maximium stress value a muscle may generate is given by the so-called intrinsic muscle strength P. For pennated muscles (angle $\alpha_{\mathrm{i}}$ between line of action and fiber direction) the maximum muscle force is given by $\mathrm{F}_{\max , \mathrm{i}}=\mathrm{P} \cdot \mathrm{A}_{\mathrm{i}} \cdot \cos \alpha_{\mathrm{i}}$.

\subsection{Rigid Body Model}

The line of action of each muscle is defined as the connection between the centroids of its origin and insertion area. In the following, the $\mathrm{x}, \mathrm{y}$-plane is chosen parallel to the Frankfurt horizontal plane with the y-axis coinciding with the axis connecting the centers of the condyles, and the x-axis directed frontally in the midsaggital plane (cf. Fig. 1, right). With the force law relating the muscle forces $F_{i}$ to the electric activities $U_{i}$, and the assumptions that (1) each joint force intersects the center of the corresponding condyle and (2) the component in direction of the condyle axis can only be transmitted by compression, the intrinsic muscle strength $\mathrm{P}$ and the joint forces can be determined using the balance of momentum. Once $\mathrm{P}$ is determined, all muscle forces follow from the force law together with the measured data.

\subsection{Finite Element Formulations}

Most chewing muscles have a complex structure, i.e., they are pennated. The muscle fibers attach to aponeuroses (cf. Fig. 3) which collect the stress gene- 


\begin{tabular}{|l|r|r|}
\hline \multirow{2}{*}{ muscle } & \multicolumn{2}{|c|}{$A_{i}\left[\mathrm{~cm}^{2}\right]$} \\
\hline masseter & \multicolumn{1}{|c|}{ right } & \multicolumn{1}{c|}{ left } \\
anterior temporal & $12.4 \pm 2.1$ & $12.4 \pm 2.5$ \\
posterior temporal & $9.4 \pm 1.4$ & $9.6 \pm 1.2$ \\
medial pterygoid & $7.2 \pm 1.2$ & $7.0 \pm 1.9$ \\
lateral pterygoid & $3.9 \pm 0.7$ & $3.6 \pm 0.7$ \\
anterior digastric & $1.5 \pm 0.5$ & $1.4 \pm 0.5$ \\
\hline
\end{tabular}

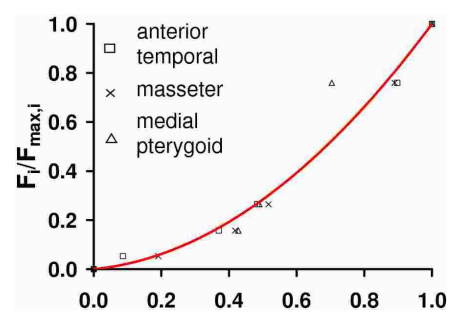

Fig. 2. Left: Physiological cross-sections $A_{i}$ averaged over the 10 test persons. Right: Force law, approximation with a second order polynomial.
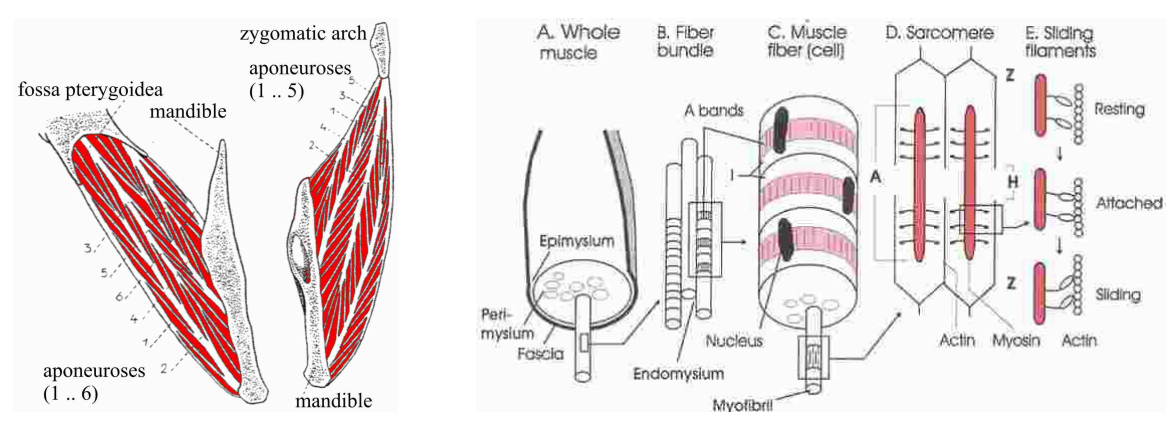

Fig. 3. Left: Morphology of pt. med. and masseter [4]. Right: Muscle physiology.

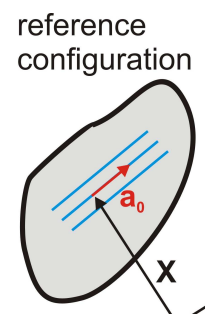

$\left|a_{0}\right|=1$ instantaneous configuration

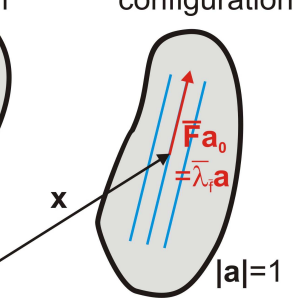

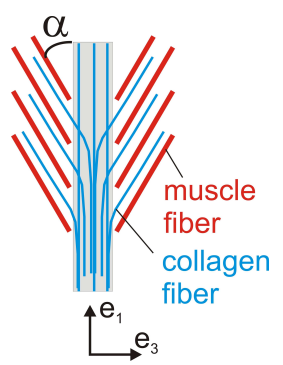

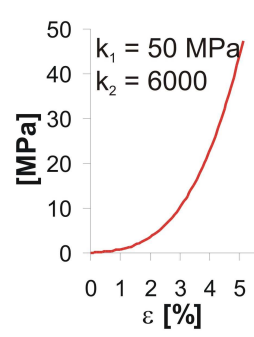

Fig. 4. Modelling and characteristics of the aponeuroses (tendinous tissue)
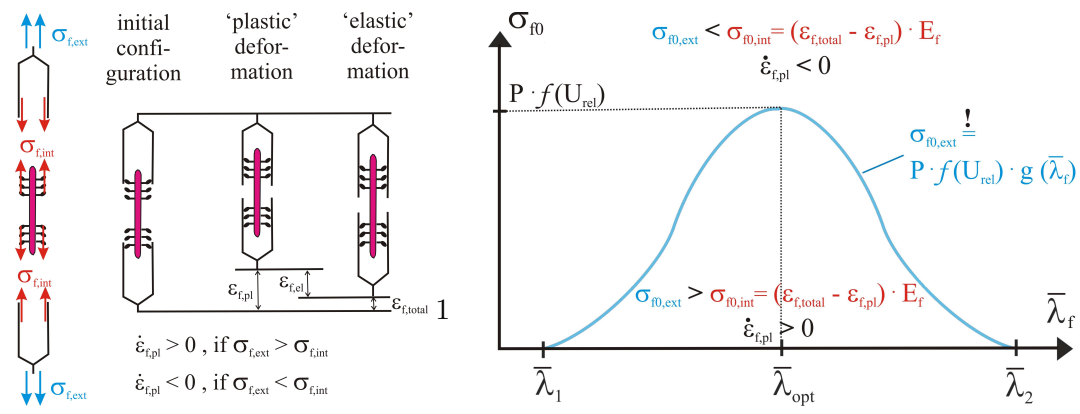

Fig. 5. Modelling and characteristics of muscle fiber contraction 
rated by the contraction of the single fibers. Therefore, to perform a realistic FE simulation, it is necessary to divide the complete muscle into contractile (muscle fibers) and tendinous tissue (aponeuroses). Both tissues consist of a soft but approximately incompressible matrix material to which fibers are added. For both tissues the matrix is modelled as a Mooney-Rivlin material. In the following, the additional contribution of the fibers is presented and implemented.

Finite tendon element: For a correct numerical implementation it is necessary to perform the well-known volumetric split of the deformation gradient which results in:

$\mathbf{F}=\left(\mathrm{J}^{1 / 3} \mathbf{I}\right) \overline{\mathbf{F}}$ with $\mathrm{J}=\operatorname{det} \mathbf{F} ; \quad \overline{\mathbf{F}}:$ modified deformation gradient

$\mathbf{C}=\mathbf{F}^{\mathrm{T}} \mathbf{F}=\mathrm{J}^{2 / 3} \overline{\mathbf{F}}^{\mathrm{T}} \overline{\mathbf{F}}=\mathrm{J}^{2 / 3} \overline{\mathbf{C}} ; \quad \overline{\mathbf{C}}$ : mod. right Cauchy-Green tensor

The free energy function for the tendinous tissue is divided into a penalty part for the dilatation and parts due to the isochoric deformation $(\overline{\mathbf{C}})$ of the matrix and fibers. Whereas the matrix material behaves isotropic, the behavior of the fiber depends additionally on a structure tensor $\mathbf{A}$ which takes into account the fiber distribution.

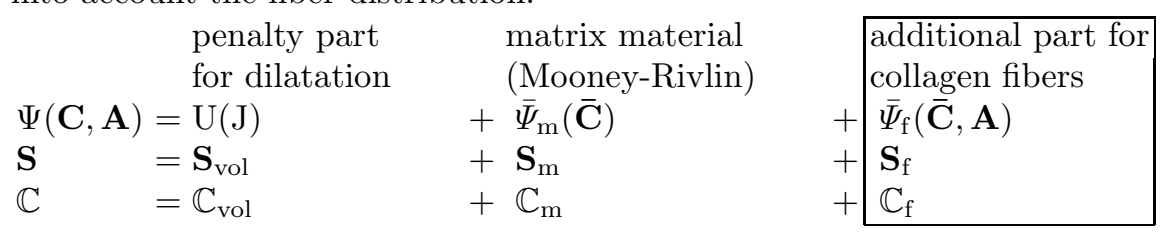

Differentiation of the free-energy function with respect to the right CauchyGreen tensor yields the 2. Piola-Kirchhoff stress tensor and the material tensor. In the following, we concentrate solely on the new contributions originating from the fibers. For the 2. Piola-Kirchhoff stresses this part is given by $\overline{\mathbf{S}}_{\mathrm{f}}=2 \partial \bar{\Psi}_{\mathrm{f}} / \partial \mathbf{C}$.

Using the fourth order projection tensor $\mathbb{P}$ the differentiation with respect to the right Cauchy-Green tensor can be replaced by the differentiation with respect to the modified right Cauchy-Green tensor [5]:

$\overline{\mathbf{S}}_{\mathrm{f}}=\mathrm{J}^{-2 / 3} \mathbb{P}:\left(2 \partial \bar{\Psi}_{\mathrm{f}} / \partial \overline{\mathbf{C}}\right)=\mathrm{J}^{-2 / 3} \mathbb{P}: \overline{\mathbf{S}}_{\mathrm{f}, \text { iso }}$

with $\mathbb{P}=\mathbb{I}-\frac{1}{3} \mathbf{C}^{-1} \otimes \mathbf{C}$ and $\mathbb{I}=\frac{\delta_{I K} \delta_{J L}+\delta_{I L} \delta_{J K}}{2}$

For the further procedure it is essential that no energy function for the fibers is introduced explicitely, but rather the contribution $\overline{\mathbf{S}}_{\mathrm{f} \text {,iso }}$ of the fibers to the 2.Piola-Kirchhoff stress tensor due to an isometric deformation is derived. Looking at the deformed configuration, the Cauchy stress of each fiber depends on the fiber stretch $\bar{\lambda}$ and is, of course, oriented in the direction a of the fiber (cf. Fig. 4). The 2. Piola-Kirchhoff stress tensor is then gained by a pull-back operation:

$\overline{\boldsymbol{\sigma}}_{\mathrm{f}}=\sigma(\bar{\lambda}) \mathbf{a} \otimes \mathbf{a}$

$\overline{\mathbf{S}_{\mathrm{f}}}=\frac{1}{\bar{\lambda}^{2}} \sigma(\bar{\lambda}) \mathbf{a}_{0} \otimes \mathbf{a}_{0} \quad$ with $\quad \lambda^{2}=\left|\overline{\mathbf{F}} \mathrm{a}_{0}\right|^{2}=\overline{\mathrm{C}}_{\mathrm{ij}} \mathrm{a}_{0}^{\mathrm{i}} \mathrm{a}_{0}^{\mathrm{j}}$ 
As suggested by Gasser, Ogden and Holzapfel [5], a density function $\rho(\varphi, \theta)$ is introduced to take into account the spatial distribution of the collagen fibers. The stress tensor is now gained by the summation of the stress tensors for all directions (unit sphere) weighed with the density function:

$\overline{\boldsymbol{S}}_{\mathrm{f}, \text { iso }}=\frac{1}{4 \pi} \int_{\varphi=0}^{2 \pi} \int_{\theta=0}^{\pi} \rho \frac{1}{\bar{\lambda}^{2}} \sigma(\bar{\lambda}) \mathbf{e} \otimes \mathbf{e} \sin \theta \mathrm{d} \theta \mathrm{d} \varphi \quad$ with $\quad \mathbf{e}=\left(\begin{array}{c}\sin \theta \cos \varphi \\ \sin \theta \sin \varphi \\ \cos \theta\end{array}\right)$

The integral of the density function over the unit sphere has to be zero, i.e., the following normalization condition has to be fulfilled:

$\frac{1}{4 \pi} \int_{\varphi=0}^{2 \pi} \int_{\theta=0}^{\pi} \rho(\varphi, \theta) \sin \theta \mathrm{d} \theta \mathrm{d} \varphi=1$

As can be seen in the scheme given in Fig. 4, the muscle fibers (pennation angle $\alpha$ ) are attached to the aponeurosis by collagen fibers. Therefore, it is assumed that all fibers lie in the interval $\frac{\pi}{2}-\alpha \leq \theta \leq \frac{\pi}{2}+\alpha$ and are distributed uniformly with repect to $\theta$. The in-plane fiber distribution $\rho(\varphi)$ of the aponeurosis has to be a $\pi$-periodic function. Therefore, the density function ( $\mathrm{N}$ follows from the normalization equation) is chosen as:

$$
\begin{gathered}
\rho(\varphi, \theta)=\left\{\begin{array}{c}
\rho(\varphi)=\frac{A_{0}}{N}+\sum_{i=1}^{n} \frac{A_{i}}{N} \cos ^{2 m_{i}}\left(\varphi-\varphi_{0, i}\right) \text { for } \theta \in\left[\frac{\pi}{2}-\alpha, \frac{\pi}{2}+\alpha\right] \\
\text { and } \mathrm{m}_{\mathrm{i}} \in\{1,2,3, \ldots\}
\end{array}\right. \\
\quad N=\sin \alpha\left(A_{0}+\sum_{i=1}^{n} A_{i} \prod_{j=1}^{m_{i}} \frac{2\left(m_{i}-j\right)+1}{2 j}\right)
\end{gathered}
$$

Collagen fibers show a quickly increasing stiffness upon elongation. At first, the curled fibers will be stretched and oriented in load direction with a small force. At the end, the fibers are straight and in parallel, and nearly no further elongation can be achieved even by high forces. This behavior can be well approximated by an exponential function. Here, because a polynomial is of advantage later on, the first two elements of a series expansion are used:

$$
\begin{aligned}
\sigma(\bar{\lambda}) & =\mathrm{k}_{1} \bar{\lambda}^{2}\left(\varepsilon+\mathrm{k}_{2} \varepsilon^{3}\right)=\mathrm{k}_{1} \bar{\lambda}^{2}\left[\frac{1}{2}\left(\bar{\lambda}^{2}-1\right)+\mathrm{k}_{2}\left(\frac{1}{2}\left(\bar{\lambda}^{2}-1\right)\right)^{3}\right] \\
& =\bar{\lambda}^{2} \mathrm{k}_{1}\left[\mathrm{k}_{2} \bar{\lambda}^{6}-3 \mathrm{k}_{2} \bar{\lambda}^{4}\left(3 \mathrm{k}_{2}+4\right) \bar{\lambda}^{2}-\left(\mathrm{k}_{2}+4\right)\right] / 8 \\
\bar{\lambda}^{2}= & \overline{\mathrm{C}}_{\mathrm{KL}} \mathrm{e}_{\mathrm{K}} \mathrm{e}_{\mathrm{L}} ; \bar{\lambda}^{4}=\overline{\mathrm{C}}_{\mathrm{KL}} \overline{\mathrm{C}}_{\mathrm{MN}} \mathrm{e}_{\mathrm{K}} \mathrm{e}_{\mathrm{L}} \mathrm{e}_{\mathrm{M}} \mathrm{e}_{\mathrm{N}} \\
\bar{\lambda}^{6}= & \overline{\mathrm{C}}_{\mathrm{KL}} \overline{\mathrm{C}}_{\mathrm{MN}} \overline{\mathrm{C}}_{\mathrm{OP}} \mathrm{e}_{\mathrm{K}} \mathrm{e}_{\mathrm{L}} \mathrm{e}_{\mathrm{M}} \mathrm{e}_{\mathrm{N}} \mathrm{e}_{\mathrm{O}} \mathrm{e}_{\mathrm{P}}
\end{aligned}
$$

A typical stress-strain-curve is depicted in Fig. 4.

Like in [5] the material tensor for the isochoric contribution is defined as:

$\overline{\mathbb{C}}_{\mathrm{f}}=\mathbb{P}: \overline{\mathbb{C}}_{\mathrm{f}, \text { iso }}: \mathbb{P}^{\mathrm{T}}+\frac{2}{3} \mathrm{~J}^{-2 / 3} \operatorname{tr}\left[\overline{\mathbf{S}}_{\mathrm{f}, \text { iso }}\right] \overline{\mathbb{P}}-\frac{2}{3}\left(\mathbf{C}^{-1} \otimes \overline{\mathbf{S}}_{\mathrm{f}}+\overline{\mathbf{S}}_{\mathrm{f}} \otimes \mathbf{C}^{-1}\right)$

with $\overline{\mathbb{P}}=\mathbb{I}_{\mathrm{C}^{-1}}-\frac{1}{3} \mathbf{C}^{-1} \otimes \mathbf{C}^{-1}$ and $\left[\mathbb{I}_{\mathrm{C}^{-1}}\right]_{\mathrm{IJKL}}=\frac{1}{2}\left(\mathrm{C}_{\mathrm{IK}}^{-1} \mathrm{C}_{\mathrm{JL}}^{-1}+\mathrm{C}_{\mathrm{IL}}^{-1} \mathrm{C}_{\mathrm{JK}}^{-1}\right)$

To take into account that fiber support exists only for fiber elongation, a 
function $\mathrm{h}(\bar{\lambda})$ is introduced which is 1 for fiber elongation and 0 for fiber shortening. Therefore, the range of values for $\varphi$ and $\theta$ (due to symmetry only half of the unit sphere has to be evaluated) is divided in $t_{1}$ and $t_{2}$ parts, respectively.

$$
\begin{aligned}
& h\left(\bar{\lambda}\left(\frac{\varphi_{r}+\varphi_{r+1}}{2}, \frac{\theta_{s}+\theta_{s+1}}{2}\right)\right)=\left\{\begin{array}{l}
1 \text { for } \bar{\lambda}\left(\frac{\varphi_{r}+\varphi_{r+1}}{2}, \frac{\theta_{s}+\theta_{s+1}}{2}\right) \geq 1 \\
0 \text { for } \bar{\lambda}\left(\frac{\varphi_{r}+\varphi_{r+1}}{2}, \frac{\theta_{s}+\theta_{s+1}}{2}\right)<1
\end{array}\right. \\
& \varphi_{r}=\frac{2 \pi}{t_{1}}(r-1) \quad, r=1,2, \ldots, \mathrm{t}_{1}
\end{aligned}
$$

Finally, for $\overline{\mathbb{C}}_{\mathrm{f}, \text { iso }}$ the expression given below is gained. The integrals depend only on the structure, and primitives can be found and implemented in an FE code.

$$
\begin{aligned}
& {\left[\overline{\mathbb{C}}_{\mathrm{f}, \text { iso }}\right]_{\mathrm{IJKL}}=2 \frac{\partial\left[\mathbf{S}_{\mathrm{f}, \text { iso }}\right]_{\mathrm{IJ}}}{\partial \overline{\mathbf{C}}_{\mathrm{KL}}}} \\
& =\frac{k_{1}}{8 \pi} \sum_{r=1}^{t_{1}} \sum_{s=1}^{t_{2}} h(\bar{\lambda}) \cdot\left\{\left(3 k_{2}+4\right) \int_{\varphi=\varphi_{r}}^{\varphi_{r+1}} \int_{\theta=\theta_{s}}^{\theta_{s+1}} \rho(\varphi) e_{I} e_{J} e_{K} e_{L} \sin \theta d \theta d \varphi-\right. \\
& \quad-\quad 3 k_{2} \bar{C}_{M N} \int_{\varphi=\varphi_{r}}^{\varphi_{r+1}} \int_{\theta=\theta_{s}}^{\theta_{s+1}} \rho(\varphi) e_{I} e_{J} e_{K} e_{L} e_{M} e_{N} \sin \theta d \theta d \varphi+ \\
& \left.\quad+k_{2} \bar{C}_{M N} \bar{C}_{O P} \int_{\varphi=\varphi_{r}}^{\varphi_{r+1}} \int_{\theta=\theta_{s}}^{\theta_{s+1}} \rho(\varphi) e_{I} e_{J} e_{K} e_{L} e_{M} e_{N} e_{O} e_{P} \sin \theta d \theta d \varphi\right\}
\end{aligned}
$$

Finite muscle element for quasi-static contraction: As mentioned above, a Mooney-Rivlin material formulation is chosen to describe the matrix, i.e., besides the penalty part for dilatation $\mathrm{U}(\mathrm{J})$ all other parts depend on isochoric deformation measures $\left(\bar{\lambda}_{\mathrm{f}}, \overline{\mathbf{F}}, \overline{\mathbf{C}}\right)$.

As can be seen in Fig. 3, a muscle fiber consists of layers of sarcomeres, which are the force generating elements of a muscle. Due to this series connection, during quasi-static loading each sarcomere layer has to generate the same force, i.e., the same mean stress value. There exists an optimal fiber stretch value $\bar{\lambda}_{\mathrm{f}, \mathrm{opt}}$ for which the highest number of cross-links between myosin and actin filaments is given and therefore the highest stress value can be achieved. The peak of this stress-stretch-curve has the value $\sigma_{\max , 0}=\mathrm{P} \cdot f\left(\mathrm{U}_{\text {rel }}\right)$ where $f\left(\mathrm{U}_{\mathrm{rel}}\right)=\mathrm{F} / \mathrm{F}_{\max }=\sigma_{\mathrm{fo}} / \sigma_{\mathrm{f} 0 \text {, max }}$ is the force law presented in chapter 2.2. The influence of the fiber stretch $\bar{\lambda}_{\mathrm{f}}$ is taken into account by the function $\mathrm{g}\left(\bar{\lambda}_{\mathrm{f}}\right)$. For quasi-static contraction the stress generated in the fiber and the stress acting externally on the fiber have to be equal. The external stress is set to the stress value according to the actual fiber stretch and electric activation: $\sigma_{\mathrm{f} 0 \text {,ext }}=\mathrm{P} \cdot f\left(\mathrm{U}_{\mathrm{rel}}\right) \cdot \mathrm{g}\left(\bar{\lambda}_{\mathrm{f}}\right)$. This stress value refers to the physiological crosssection in the undeformed state. The corresponding Cauchy stress is then 
$\sigma_{\mathrm{f}, \text { ext }}=\sigma_{\mathrm{f} 0 \text {,ext }} \cdot \mathrm{dA} / \mathrm{da}=\bar{\lambda}_{\mathrm{f}} \sigma_{\mathrm{f} 0 \text {,ext }}$. The internal stress value depends on the elastic deformation of the fiber (elastic deformation of the filaments) which is very small in comparison with the 'plastic' deformation which is given by the relative movement of actin and myosin filaments (cf. Fig. 5). In contrast to the tendinous tissue, the muscle fibers of the contractile tissue are locally oriented in parallel, i.e., there exists only one fiber direction. As long as passive behavior is of no interest and therefore not modelled (muscle fibers are surrounded by thin layers of tendinous tissue, which impede a elongation of the fibers), the active fiber stresses are added to the right hand side and no additional entries due to the fibers occur in the stiffness matrix. Using the plastic fiber deformation at each Gauss-point as history variable, the muscle deformation corresponding to the actual electric activation can be found.

\section{Results and Dicussion}

The following results arise from the rigid body analysis based on a purely geometrical estimation of the lines of action.

For the intrinsic muscle strength a mean value $\mathrm{P}=0.32 \pm 0.12 \mathrm{~N} / \mathrm{mm}^{2}$ was found. The results for the muscle and joint forces under a resultant bite force magnitude of $150 \mathrm{~N}$ are shown in Fig. 6. Here, corresponding muscle and joint forces of the right and left side have been averaged. The right muscles and the right condyle perform the same task for $\varphi=0^{\circ}, 60^{\circ}, 90^{\circ}, 180^{\circ}, 270^{\circ}$, $300^{\circ}$ as the left muscles and the left condyle for $\varphi=0^{\circ}, 300^{\circ}, 270^{\circ}, 180^{\circ}$, $90^{\circ}, 60^{\circ}$, respectively. The values for the muscle volumes calculated from the

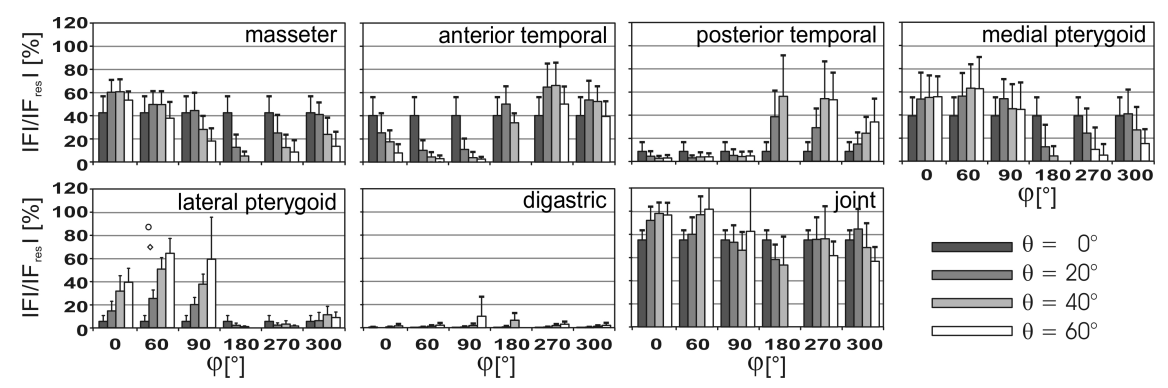

Fig. 6. Muscle and joint forces of the 'right' side (values of right and left side are averaged for corresponding tasks) under a resultant bite force of $F_{\text {res }}=150 \mathrm{~N}$.

MRTs and the correlating physiological cross-sectional areas correspond well with those found in literature $[3,6]$. This holds especially for test persons of about the same age quoted in [6].

The estimation of the lines of action based on the geometry delivers reliable results if the individual muscle is activated homogeneously. However, especially for laterally and medially oriented tasks, measurements show a 
heterogeneous activation of the musculature as also described in [7]. This muscle behavior might essentially influence the lines of action. Therefore, the presented results for the intrinsic muscle strength $\mathrm{P}$ are presumably less accurate for these tasks than for the protrusive or vertical (symmetric) tasks. Nevertheless, the mean value for all calculated intrinsic muscle strengths corresponds well with values given in literature. For example, Weijs and Hillen [3] found $\mathrm{P}=0.37 \mathrm{~N} / \mathrm{mm}^{2}$.

With the exception of the anterior temporal, the individual muscles developed the highest force values in clenching directions which corresponded roughly to their line of action. This supports the assumption that the motor control selects the activation state of the masticatory muscles with regard to their directional effectiveness. The relatively high force generation of the anterior temporal during lateral and posterior force development, however, might be essential for stabilizing the ipsilateral jaw joint during these tasks.

The joint force magnitude ranges from about $60 \%$ to $100 \%$ of the magnitude of the resultant bite force. It is known from measurements that chewing forces lie between 50 and $250 \mathrm{~N}$, i.e., $\mathrm{F}_{\text {res }}=150 \mathrm{~N}$ corresponds to a moderate chewing force. However, maximum forces with magnitudes over $800 \mathrm{~N}$ can be generated.

\section{Conclusions and Outlook}

A reliable calculation of the intrinsic muscle strength $P$ requires an individual adjustment of the force law. The presented force and EMG measurements clearly show a non-linear dependence of the muscle force on the electric activity. Motor control seems to favor a directional effectiveness of the muscles when selecting the task-dependent intermuscular activation patterns. When large bite forces are developed, the joint force magnitudes are about $60 \%$ of the magnitude of the resultant bite force. This might predispose an overloading of the jaw joint tissues.

With the developed finite elements for tendinous and contractile tissue, additionally the contraction of the muscles under inhomogeneous electric activation can be taken into account, and the lines of action can be computed for every motor task. This will yield more reliable results for the intrinsic muscle strength $\mathrm{P}$ and the muscle forces. Furthermore, the FE analysis will give detailed information about the stresses in the joint region, i.e., in the articular disc and fossa mandibulae. 



\section{Bibliography}

[1] Schindler, H.J., Rues, S., Türp, J.C., Lenz, J.: Activity patterns of the masticatory muscles during feedback-controlled simulated clenching activities. Eur. J. Oral Sci. 113 (2005), 469-478.

[2] van Eijden, T.M.G.J., Korfage, J.A.M., Brugman, P.: Architecture of the human jaw-closing and jaw-opening muscles. The Anatomical Record 248 (1997), 464-474.

[3] Weijs, W.A., Hillen, B. : Cross-sectional areas and estimated intrinsic strength of the human jaw muscles. Acta morph. nederl.-scand $\mathbf{2 3}$ (1985), 267-274.

[4] Schumacher, G.H.: Funktionelle Morphologie der Kaumuskulatur. VEB Gustav Fischer Verlag, Jena 1961.

[5] Gasser, T.C., Ogden, R.W., Holzapfel, G.A.: Hyperelastic Modelling of arterial layers with distributed collagen fibre orientations. J. R. Soc. Interface 3 (2006), 15-35.

[6] C.W. Hsu, Y.Y. Shiau, C.M. Chen, K.C. Chen, H.M. Liu: Measurement of the size and orientation of human masseter and medial pterygoid muscles. Proc. Natl. Sci. Counc. ROC(B), Vol. 251 (2001), 45-49.

[7] Blanksma, N.G., van Eijden, T.M.G.J.: Electromyographic hetrogeneity in the human temporalis and masseter during static biting, open/close excursions, and chewing. J. Dent. Res. 74 (1995), 13181327. 
